\title{
Estudio bibliométrico de la literatura sobre el libro antiguo en la base de datos ISOC
}

\author{
Antonio Pulgarín Guerrero \\ José Luis Herrera Morillas \\ María Josefa Rosado Pacheco *
}

Artículo recibido:

27 de agosto de 2012.

Artículo aceptado:

16 de noviembre de 2012.

\section{RESUMEN}

Se recopilan 907 trabajos relacionados con libros antiguos incluidos en la base de datos ISOC, para realizar un estudio bibliométrico (860 artículos de revistas y 47 colaboraciones presentadas a congresos). Se establece el siguiente esquema de estudio para analizar los trabajos localizados: distribución por tipo de publicación, por materias, por años de publicación, por nombre de las revistas, por autores; también se establece la dispersión de la literatura, la productividad científica de los autores y las características de las revistas según se indica en RESH (Revistas Españolas de Ciencias Sociales y Humanidades).

* Los tres autores pertenecen a la Universidad de Extremadura, España. (Antonio: pulgarin@unex.es); (José Luis: jlhermor@unex.es); (María Josefa: mjrospac@ alcazaba.unex.es).

INVESTIGACIÓN BIBLIOTECOLÓGICA, Vol. 27, Núm. 59, enero/abril, 2013, México, ISSN: 0187-358X. pp. 121-140 
Palabras clave: Base de datos ISOC; Estudio bibliométrico; Libros antiguos.

ABSTRACT

A biblometric study of the literature on old books in the ISOC database

Antonio Pulgarin-Guerrero, José Luis Herrera-Morillas and María Josefa Rosado-Pacheco

A bibliometric study describes 907 works on old books included in the ISOC database (860 journal articles and 47 papers presented at conferences). The study analyzed distribution by type and year of publication, subject, journal name, and authors. The diffusion of the literature, the authors' scientific productivity and the characteristics of the journals as indicated in RESH are also described.

Keywords: ISOC database; Bibliometric Study; Old book.

\section{INTRODUCCIÓN}

Ta aplicación de las técnicas y métodos de la bibliometría al estudio de Llas colecciones de libros antiguos es un tema de interés que presenta un panorama de investigación apenas desarrollado. La investigación existente, anterior a este trabajo, ha sido abordada a través de una serie de trabajos desde enfoques distintos.

Entre los trabajos de investigación relacionados con el tema se pueden citar como más característicos los de Aguirre (1987), Herrero (1997), Pulgarín, Herrera y Marroquín (2007) o el de Pulgarín y Herrera (2009), además de otros relacionados con el estudio de publicaciones antiguas de medicina, como los de Puche (1975), Olagüe (1976), Guillén et al. (1988), Sillero (1992); y de farmacia, como los de Sánchez (1978) o de Mansilla (1984).

El trabajo de Aguirre (1987) se centra en la literatura española del Siglo de Oro y se observa en el título la expresión bibliometría con el adjetivo "elemental". Analiza la producción bibliográfica de obras literarias del siglo XVII y destaca la proporción de los diferentes géneros.

El estudio de Herrero (1997) consiste en el diseño y creación de una base de datos para la descripción de una colección de libros antiguos del siglo XVI. 
El aspecto bibliométrico se reduce a la elaboración (a modo de resultados) de unos gráficos con los datos cuantitativos de las obras analizadas, según los lugares de impresión, la fecha de publicación, la procedencia de los autores, la lengua de publicación, los tipos de escritura, las encuadernaciones y las materias.

En la publicación de Pulgarín, Herrera y Marroquín (2007) se estudia y difunde, de manera global, el fondo antiguo de la Biblioteca de la Real Sociedad Económica Extremeña de Amigos del País (RSEEAP). Para ello se lleva a cabo la recopilación de los datos bibliográficos del fondo correspondientes a los siglos XVI-XVIII. Estos datos son organizados para su posterior tratamiento y análisis bibliométrico y se cotejan con el Catálogo Colectivo del Patrimonio Bibliográfico Español (CCPBE), con el propósito de observar la mayor o menor singularidad o rareza del fondo (los datos de la Biblioteca de la RSEEAP no estaban recogidos en el CCPBE); y analizan la evolución cronológica del número de libros, la productividad de los autores, el lugar de edición y la distribución de libros por materias y por editores-impresores.

Del trabajo de Pulgarín y Herrera (2009) habría que destacar la definición de unas posibles vías de investigación que pueden orientar la aplicación de la bibliometría al estudio de las colecciones de fondo antiguo conservadas en España. Estas vías se concretan en torno a tres ejes: 1. Colecciones de bibliotecas; 2. Bases de datos y catálogos colectivos especializados; 3 . Revistas científicas especializadas y afines. De cada una de ellas se establecen las cualidades que definen el objeto de estudio y se establece un listado de ejemplos al que se podrían aplicar las investigaciones; y también, los indicadores bibliométricos más propios para utilizar en cada caso.

Respecto a las investigaciones en medicina y en farmacia, la mayoría de ellas está vinculada a la Universidad de Valencia, pionera en la introducción en España de los estudios bibliométricos y en el desarrollo de la documentación médica, y desarrollan desde la década de 1970 una activa labor en el campo de la bibliometría, a partir de los trabajos del grupo dirigido por el profesor López Piñero.

Otro tipo de trabajos que guardan cierta relación son los que se basan totalmente o en parte en el estudio de una determinada producción bibliográfica y/o editorial, y que suelen incorporar en su metodología algún tipo de análisis estadístico, por ejemplo, en el trabajo de Sáez y Marset (1993) se realiza un análisis de la producción científica en medicina en Murcia a lo largo del siglo XVIII con el fin de evaluar el nivel alcanzado. Pero la mayoría de este tipo de trabajos son más bien de carácter histórico y/o bibliográfico, como, por ejemplo, los trabajos de Fernández (1984); Berger (1988); Delgado y Cordón (1990); Liñán (1992); Pardo (1997); Gil (2004); García (2005) o de Suárez (2005). 
En un diseño muy similar hay que destacar del grupo de trabajos de investigación denominado tipobibliografías o repertorios tipobibliográficos aquellos que se han realizado en función de una de las características intrínsecas de las obras: su lugar de publicación. En ellos se trata de reunir, analizar y descubrir las obras que han sido impresas en un determinado lugar, ya sea una localidad, provincia, región o nación.

El objetivo general del estudio es recopilar artículos de revistas y comunicaciones presentados en congresos relacionados con libros antiguos que se incluyen en la base de datos ISOC y llevar a cabo un análisis bibliométrico de la información resultante.

Los objetivos específicos son:

- Indagar y trazar el estado de la cuestión de la investigación sobre el libro antiguo en España mediante indicadores bibliométricos.

- Aplicar las técnicas y métodos de la bibliometría al estudio de las publicaciones recopiladas sobre libros antiguos, fundamentalmente modelos matemáticos y estadísticos.

- Diseñar una base de datos mediante el empleo de un gestor bibliográfico para facilitar el tratamiento de la información recopilada.

- Trazar una visión de conjunto sobre la investigación del libro antiguo en España, a partir del análisis de la información recopilada.

- Evaluar y tener una visión más exacta de la base de datos ISOC.

\section{Metodología}

En la metodología aplicada destacan dos aspectos. En primer lugar la localización y recuperación de las referencias de los trabajos sobre libros antiguos recogidos en la Base de Datos ISOC <http://bddoc.csic.es:8080/>.

En segundo lugar la aplicación de las herramientas metodológicas propias de la bibliometría para analizar los datos obtenidos de la base de datos.

La base de datos ISOC-Ciencias Sociales y Humanidades es una base de datos referencial y bibliográfica que fundamentalmente recoge referencias de artículos publicados en revistas españolas del ámbito de las ciencias sociales y humanas. Esta base de datos es producida por el Centro de Ciencias Humanas y Sociales (CCHS) del CSIC.

En cuanto a su cobertura temática cubre las siguientes áreas: Antropología, Arqueología, Bellas Artes, Biblioteconomía y Documentación, Ciencias Políticas, Derecho, Economía, Educación, Filosofía, Geografía, Historia, Lingüística, Literatura, Psicología, Sociología, Urbanismo y América Latina. 
Las fuentes que contiene son 2.330 publicaciones periódicas editadas en España, especializadas en las áreas antes mencionadas, además de monografías, actas de congresos, informes y tesinas. Cuenta con un volumen de 650.412 referencias bibliográficas $95 \%$ del cual corresponde a artículos publicados en revistas); 328.222 registros con afiliación institucional de los autores; 223.335 registros con resumen de autor; y 129.472 enlaces al texto completo de artículos disponibles en acceso abierto. ${ }^{1}$

Las búsquedas se pueden hacer de dos formas: consultando la base de datos completa o por áreas temáticas.

Para llevar a cabo la localización y recuperación de las referencias lo primero que se hizo fue elaborar una lista de términos para emplearlos en las búsquedas. El principio que ha guiado la selección de estos términos ha sido reflejar los contenidos más característicos del estudio e investigación del libro antiguo en nuestro país (se han tenido en cuenta los temarios de las asignaturas de los planes de estudio de Documentación y los índices de los manuales más representativos), pretendiendo la exhaustividad en la recuperación y siendo conscientes de que en una primera etapa de la búsqueda se iban a recuperar documentos no pertinentes; valía la pena entonces, hacer esto en aras de la máxima exhaustividad. Dichos términos son: libro, impreso, manuscrito, códice, biblioteca, colección, fondo, edición, imprenta, impresor, editor, librero, tipobibliografía, encuadernación, grabador, grabado, estampa, ilustración, patrimonio bibliográfico. Se han empleado en singular y en plural (tras comprobar que los términos en singular, con frecuencia, no recuperaban aquellos que incluían la misma palabra en plural).

Las búsquedas se han realizado, fundamentalmente, a través del campo de título accediendo a la base de datos en línea, y se han llevado a cabo durante los meses de marzo y abril de 2012. La estrategia de búsqueda ha consistido en localizar los registros con los términos antes mencionados, y a continuación se han visualizado los registros y se han eliminado las referencias inadecuadas, que han sido numerosas, pues como ya se explicó se ha querido favorecer la exhaustividad.

Los registros recuperados de la base de datos contienen estos campos: autor, título, ISSN, revista, datos de la fuente, lugar de trabajo, descriptores y resumen.

Para trabajar con los datos obtenidos de las búsquedas y facilitar el posterior análisis de ellos, se diseñó una base de datos que permitiera almacenar la información. Esta base de datos se ha creado con el gestor bibliográfico personal en línea RefWorks <http://www.refworks.com/es/>.

1 Datos indicados en la web de la base de datos, que señalan como fecha de éstos el 2 de enero de 2012. 
Este gestor bibliográfico cuenta con 67 campos diferentes de los que hemos seleccionado los siguientes:

- Tipo de referencia (acta de conferencia, artículo de revista, etcétera).

- Autores.

- Título.

- Publicación completa (para artículos introducimos aquí el título de la revista).

- Páginas.

- Descriptores.

- Resumen.

- Afiliación del autor.

- ISSN o ISBN.

A partir de los datos obtenidos hemos elaborado una serie de tablas para agrupar los datos y facilitar su estudio (tipo de publicación, materias, año de publicación, revistas, autores, afiliación de los autores).

En la distribución por el tipo de publicación se distinguen artículos de revista y comunicaciones a congresos.

Para la distribución por materias se definieron 10 categorías que representan grandes áreas temáticas (inspiradas en los términos empleados para la realización de las búsquedas):

1. Colecciones. Bibliotecas.

2. Comercio y tasación.

3. Encuadernación.

4. Historia del libro.

5. Ilustración y decoración.

6. Imprenta.

7. Impresores. Editores. Libreros.

8. Impresos. Ediciones y estudios.

9. Manuscritos. Códices.

10. Patrimonio bibliográfico. Conservación y difusión.

A cada documento se le ha asignado sólo una categoría, de tal modo que cuando el contenido de un documento está relacionado con dos o más de éstas, se ha optado por atribuirle la que mejor lo representa.

La distribución de documentos por años de publicación se completa con una tabla en la que se dividen los años de publicación en intervalos de 5 años, para reflejar el número de documentos publicado en cada quinquenio. 
La distribución de los artículos por revistas se indica en una tabla en la que aparece un listado alfabético con las revistas más productivas y el número de artículos publicados en cada una. En otra, se reproducen los indicadores asignados por RESH a cada una de estas revistas. RESH es un sistema de información que integra indicadores de calidad para las revistas de Ciencias sociales y Humanidades <http://epuc.cchs.csic.es/resh/>.

Para precisar la afiliación de los autores se ha buscado su lugar de trabajo y se ha creado una tabla en Excel con tales lugares y con el número de autores que trabajan en cada institución.

\section{RESULTADOS Y DISCUSIÓN}

\section{Distribución de los documentos por el tipo de publicación}

El número total de documentos recuperados de la base de datos ISOC ha sido de 907 . De éstos, 860 corresponden a artículos y 47 a comunicaciones presentadas en congresos. Estos datos son comprensibles, en primer lugar debido a que la base de datos se alimenta fundamentalmente de revistas, y en segundo porque los artículos son la publicación por excelencia de los investigadores en gran parte de las disciplinas, ya que permiten recoger trabajos de mayor envergadura y de amplio contenido, características propias, con frecuencia, de las investigaciones relacionadas con los libros antiguos.

\section{Distribución de los documentos por materias}

En la Tabla 1 se presentan los 907 documentos distribuidos por materias. Para ello, se tuvieron en cuenta las 10 categorías definidas al inicio del trabajo. Aparecen en orden de mayor a menor número de documentos, indicando también el porcentaje.

Tabla 1. Distribución de documentos por materias

\begin{tabular}{|l|c|c|}
\hline \multicolumn{1}{|c|}{ Materias } & Núm. de documentos & $\%$ \\
\hline Colecciones. Bibliotecas & 252 & 27,78 \\
\hline Ilustración y decoración & 144 & 15,88 \\
\hline Manuscritos. Códices & 130 & 14,33 \\
\hline Impresos. Ediciones y Estudios & 81 & 8,93 \\
\hline Historia del libro & 76 & 8,38 \\
\hline Imprenta & 72 & 7,94 \\
\hline Impresores. Editores. Libreros & 51 & 5,62 \\
\hline
\end{tabular}




\begin{tabular}{|l|c|c|}
\hline Patrimonio bibliográfico. Conservación y Difusión & 49 & 5,40 \\
\hline Comercio y tasación & 29 & 3,20 \\
\hline Encuadernación & 23 & 2,54 \\
\hline Total & 907 & 100 \\
\hline
\end{tabular}

La categoría que destaca con más documentos es "Colecciones. Bibliotecas" (252 documentos, el 27,75 \%). Los trabajos incluidos bajo esta denominación suelen estar dedicados, por ejemplo, al estudio de los libros de una determinada biblioteca; a aquellos que forman parte de la colección de un bibliógrafo; o al estudio de impresos de un periodo cronológico concreto, etcétera.

A continuación le siguen dos categorías ("Ilustración y decoración"; "Manuscritos. Códices") con cifras muy parecidas (144 y 130 documentos). Los trabajos sobre "Ilustración y decoración" se centran en el estudio de las ilustraciones como una parte formal del libro, prestando más o menos atención, según los casos, a su dimensión y análisis artístico. Los "Manuscritos. Códices" abordan la recopilación y el estudio de esta tipología documental, con especial atención a los estudios codicológicos de ejemplares concretos, como los numerosos trabajos de Juan José Batalla Rosado, uno de los autores más productivos.

Con unos valores en torno al 8 o 9\%, aparecen tres categorías: "Impresos. Ediciones y Estudios"; "Historia del libro" e "Imprenta”. Dentro de la primera se engloban trabajos muy variados, desde los dedicados a ejemplares concretos, a los libros impresos en general, o a impresos representativos de un determinado tema o materia. La segunda categoría aúna estudios de aspectos variados sobre el libro antiguo, siempre desde un punto de vista histórico. En la tercera, están representadas las investigaciones sobre la imprenta y sus diferentes aspectos, y en éstos por ejemplo son característicos los dedicados a la imprenta de alguna ciudad concreta donde se engloban las tipobibliografías.

Las categorías "Impresores. Editores. Libreros" y "Patrimonio bibliográfico. Conservación y difusión" se acercan, cada una, al 6\%. Los trabajos sobre "Impresores" suelen tratar del papel de los impresores en la edición de los libros, sobre todo a partir de la segunda mitad del siglo XVI, o son estudios que tratan sobre algún impresor concreto. "Patrimonio bibliográfico. Conservación y difusión" abarca trabajos sobre legislación y sobre restauración y conservación; y también sobre las nuevas tecnologías aplicadas a facilitar el acceso, la conservación y la difusión de los libros antiguos.

En último lugar están presentes las categorías "Comercio y tasación” y "Encuadernación” (con el 3,19 \% y el 2,53 \%, respectivamente). En aquélla 
los libros más recientes están dedicados a la tasación como proceso de tratamiento documental del libro antiguo y la mayoría se centra en el comercio, con trabajos de carácter histórico. Los artículos que tratan a la "Encuadernación" con frecuencia son estudios acerca de las encuadernaciones de los inicios de la imprenta en España o de alguna biblioteca o colección en particular, o sobre algún otro estilo.

\section{Distribución de los documentos por años de publicación}

Destacan los años 1997, 2000, 2001, 2009, y 2010 con 38, 36, 36, 38 y 36 documentos, respectivamente; seguidos de los años 1992, 1995 y 2002 con 34, 33 y 33 documentos. Los años con pocas publicaciones se distribuyen de forma muy dispersa entre un rango muy amplio de años.

Si los documentos son agrupados por periodos de 5 años (Figura 1), se observa un progresivo incremento hasta el quinquenio 1991/1995, con los valores más altos (169 documentos), y donde este último presenta un crecimiento de más del doble respecto al anterior. Los siguientes quinquenios mantienen cifras altas y muy parecidas, aunque van descendiendo ligeramente.

Esta tendencia es fácilmente justificable, pues las publicaciones crecen con la investigación y ésta va muy ligada a la sociedad del conocimiento y al desarrollo de las universidades, y ambos factores se han ido incrementando en nuestro país con el paso de los años y especialmente en las últimas décadas.

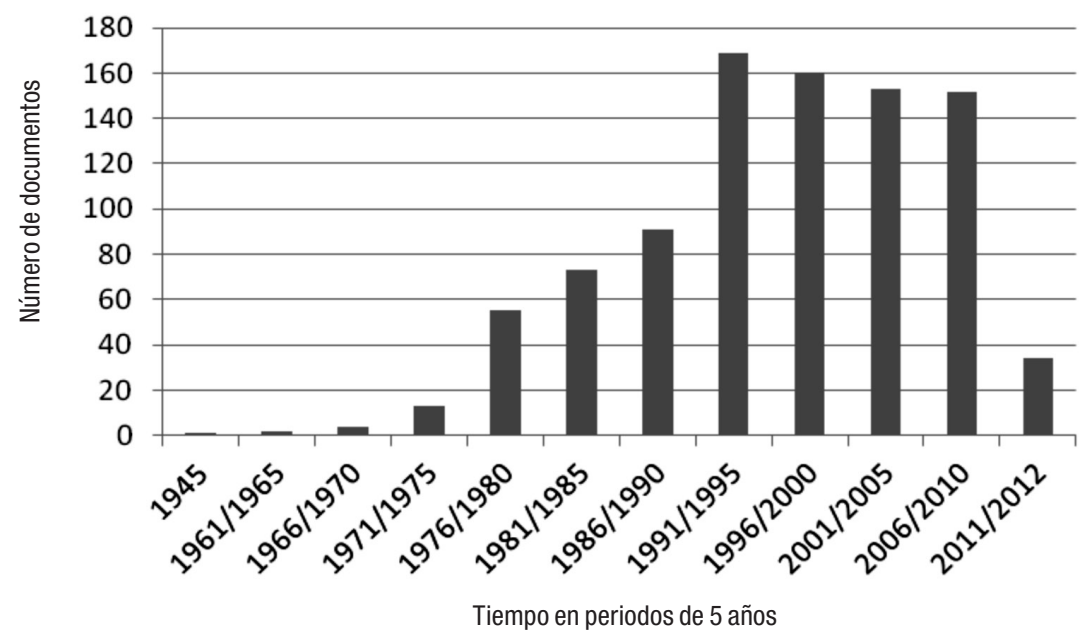

Figura 1. Distribución de los documentos por periodos de cinco años 


\section{Distribución de los artículos por revistas}

Los 860 artículos recuperados han sido publicados en un total de 291 revistas diferentes. Esto supone una productividad media de casi 3 artículos por revista $(2,955)$, lo que hay que entender como una productividad baja o una alta dispersión de la literatura. Se han seleccionado 15 revistas con una productividad mayor o igual a 10 artículos publicados. Las revistas seleccionadas, todas españolas, se muestran en la Tabla 2. En la tabla se incluye información sobre el editor y la materia (asignada por Dialnet).

De las 15 revistas, 5 están relacionadas con el área de Biblioteconomía y Documentación (Anales de Documentación; Boletín de la ANABAD; Boletín de la Asociación Andaluza de Bibliotecarios; Revista General de Información y Documentación; Pecia Complutense); 4 con Historia; 2 con Estudios locales; 2 con Arte; 1 con Antropología y 1 con Filología.

Tabla 2. Revistas más productivas ( $\geq 10$ artículos)

\begin{tabular}{|c|c|c|c|}
\hline Nombre de la revista & Editor & $\begin{array}{l}\text { Materias asignada } \\
\text { por Dialnet }\end{array}$ & $\begin{array}{l}\text { Núm. } \\
\text { artículos }\end{array}$ \\
\hline Reales Sitios & Patrimonio Nacional & $\begin{array}{l}\text { Arte: Historia del arte. } \\
\text { Artes plásticas }\end{array}$ & 28 \\
\hline $\begin{array}{l}\text { Revista General de Información } \\
\text { y Documentación }\end{array}$ & $\begin{array}{l}\text { Universidad Complutense. } \\
\text { Facultad de Documentación }\end{array}$ & $\begin{array}{l}\text { Ciencias sociales: Información } \\
\text { y Documentación }\end{array}$ & 23 \\
\hline Boletín de la ANABAD & $\begin{array}{l}\text { Confederación de } \\
\text { Asociaciones de Archiveros, } \\
\text { Bibliotecarios, Museólogos y } \\
\text { Documentalistas. ANABAD }\end{array}$ & $\begin{array}{l}\text { Ciencias sociales: Información } \\
\text { y Documentación }\end{array}$ & 19 \\
\hline $\begin{array}{l}\text { Revista Española de } \\
\text { Antropología Americana }\end{array}$ & $\begin{array}{l}\text { Universidad Complutense. } \\
\text { Departamento de Historia de } \\
\text { América II (Antropología de } \\
\text { América) }\end{array}$ & $\begin{array}{l}\text { Ciencias sociales: Antropolo- } \\
\text { gía. Etnología }\end{array}$ & 16 \\
\hline $\begin{array}{l}\text { Cuadernos de Arte e Icono- } \\
\text { grafía }\end{array}$ & $\begin{array}{l}\text { Fundación Universitaria } \\
\text { Española: Seminario de arte } \\
\text { Marqués de Lozoya }\end{array}$ & $\begin{array}{l}\text { Arte: Historia del arte. Artes } \\
\text { plásticas }\end{array}$ & 15 \\
\hline $\begin{array}{l}\text { Anales del Instituto de Estudios } \\
\text { Madrileños }\end{array}$ & $\begin{array}{l}\text { Instituto de Estudios } \\
\text { Madrileños }\end{array}$ & $\begin{array}{l}\text { Humanidades: Estudios } \\
\text { regionales y locales }\end{array}$ & 14 \\
\hline Archivo Hispalense & $\begin{array}{l}\text { Diputación Provincial de } \\
\text { Sevilla }\end{array}$ & $\begin{array}{l}\text { Humanidades: Estudios } \\
\text { regionales y locales }\end{array}$ & 14 \\
\hline Pecia Complutense & $\begin{array}{l}\text { Universidad Complutense. } \\
\text { Biblioteca Histórica "Marqués } \\
\text { de Valdecilla" }\end{array}$ & $\begin{array}{l}\text { Ciencias sociales: Información } \\
\text { y Documentación }\end{array}$ & 14 \\
\hline $\begin{array}{l}\text { Boletín de la Asociación } \\
\text { Andaluza de Bibliotecarios }\end{array}$ & $\begin{array}{l}\text { Asociación Andaluza de } \\
\text { Bibliotecarios }\end{array}$ & $\begin{array}{l}\text { Ciencias sociales: Información } \\
\text { y Documentación }\end{array}$ & 13 \\
\hline $\begin{array}{l}\text { Al-Basit. Revista de } \\
\text { estudios albacetenses }\end{array}$ & $\begin{array}{l}\text { Instituto de Estudios Albace- } \\
\text { tenses "Don Juan Manuel" }\end{array}$ & $\begin{array}{l}\text { Historia. Instituciones. } \\
\text { Documentos }\end{array}$ & 12 \\
\hline
\end{tabular}




\begin{tabular}{|c|c|c|c|}
\hline Anales de Documentación & $\begin{array}{l}\text { Universidad de Murcia. } \\
\text { Facultad de Ciencias de la } \\
\text { Documentación }\end{array}$ & $\begin{array}{l}\text { Ciencias sociales: Información } \\
\text { y Documentación }\end{array}$ & 12 \\
\hline $\begin{array}{l}\text { Historia. Instituciones. Docu- } \\
\text { mentos }\end{array}$ & $\begin{array}{l}\text { Universidad de Sevilla. } \\
\text { Departamento de Historia } \\
\text { Medieval y Ciencias y Técnicas } \\
\text { Historiográficas }\end{array}$ & $\begin{array}{l}\text { Historia. Instituciones. } \\
\text { Documentos } \\
\text { Ciencias jurídicas: Dcho. } \\
\text { romano, } \mathrm{H}^{\mathrm{a}} \text { y Fa del derecho }\end{array}$ & 12 \\
\hline Edad de Oro & $\begin{array}{l}\text { Universidad Autónoma de Ma- } \\
\text { drid. Servicio de publicaciones }\end{array}$ & $\begin{array}{l}\text { Filologías: Filologías } \\
\text { hispánicas }\end{array}$ & 11 \\
\hline Hispania Sacra & $\begin{array}{l}\text { Consejo Superior de Investi- } \\
\text { gaciones Científicas: Instituto } \\
\text { de Historia }\end{array}$ & Humanidades: Historia & 10 \\
\hline Memoria Ecclesiae & $\begin{array}{l}\text { Asociación de Archiveros de la } \\
\text { Iglesia en España }\end{array}$ & $\begin{array}{l}\text { Humanidades: Religión. } \mathrm{H}^{\mathrm{a}} \text { de } \\
\text { las religiones }\end{array}$ & 10 \\
\hline
\end{tabular}

Estas revistas se han buscado en RESH, que aporta los resultados del análisis de las Revistas Españolas de Ciencias Sociales y Humanidades desde el punto de vista de su calidad. En la Tabla 3 se incluyen los indicadores que le asigna RESH a 14 revistas, ya que Memoria Ecclesiae no aparece en RESH. Destacan, como mejor valoradas por su impacto, la Revista Española de Antropología Americana; la revista Historia. Instituciones. Documentos; Hispania Sacra o la Revista General de Información y Documentación.

Tabla 3: Revistas más productivas con los Indicadores de RESH

\begin{tabular}{|c|c|c|c|c|c|c|}
\hline \multirow[t]{2}{*}{ Revista } & \multicolumn{3}{|c|}{$\begin{array}{c}\text { Criterios de calidad } \\
\text { editorial }\end{array}$} & \multirow{2}{*}{\begin{tabular}{|c|} 
Difusión \\
BASES DE DATOS
\end{tabular}} & \multirow{2}{*}{$\begin{array}{c}\begin{array}{c}\text { Opinión } \\
\text { expertos }\end{array} \\
2009\end{array}$} & \multirow{2}{*}{$\begin{array}{c}\text { Impacto } \\
2004-2008\end{array}$} \\
\hline & CNEAI & ANECA & LATINDEX & & & \\
\hline Reales Sitios & 6 & 9 & 20 & 7 & 3.21 & 0.110 \\
\hline $\begin{array}{l}\text { Revista General de } \\
\text { Información y } \\
\text { Documentación }\end{array}$ & 16 & 19 & 32 & 4 & 4.41 & 0.155 \\
\hline Boletín de la $A N A B A D$ & 4 & 5 & 18 & 1 & 1.69 & 0.064 \\
\hline $\begin{array}{l}\text { Revista Española de } \\
\text { Antropología Americana }\end{array}$ & 15 & 17 & 32 & 9 & 5.61 & 0.260 \\
\hline $\begin{array}{l}\text { Cuadernos de Arte e } \\
\text { Iconografía }\end{array}$ & 4 & 6 & 17 & 3 & 1.51 & 0.149 \\
\hline $\begin{array}{l}\text { Anales del Instituto de } \\
\text { Estudios Madrileños }\end{array}$ & 10 & 13 & 30 & 2 & & 0.057 \\
\hline Archivo Hispalense & 12 & 14 & 29 & 1 & 0.03 & 0.020 \\
\hline Pecia Complutense & 12 & 14 & 30 & 2 & & 0.000 \\
\hline $\begin{array}{l}\text { Boletín de la Asociación } \\
\text { Andaluza de Bibliote- } \\
\text { carios }\end{array}$ & 7 & 9 & 27 & 4 & & 0.000 \\
\hline Al-Basit & 5 & 7 & 27 & 1 & & 0.000 \\
\hline
\end{tabular}




\begin{tabular}{|l|c|c|c|c|c|c|}
\hline $\begin{array}{l}\text { Anales de } \\
\text { Documentación }\end{array}$ & 15 & 18 & 33 & 5 & 2.69 & \\
\hline $\begin{array}{l}\text { Historia. Instituciones. } \\
\text { Documentos }\end{array}$ & 11 & 13 & 26 & 3 & 4.49 & 0.225 \\
\hline Edad de Oro & 7 & 10 & 22 & 5 & 2.35 & 0.162 \\
\hline Hispania Sacra & 17 & 20 & 33 & 13 & 7.17 & 0.202 \\
\hline Memoria Ecclesiae & --- & --- & --- & --- & -- & - \\
\hline
\end{tabular}

También se ha elaborado la distribución total de revistas por áreas temáticas, según asigna RESH (Tabla 4). Se observa que el área correspondiente a Historia es la que mayor número de revistas contiene, seguida a una distancia considerable por el área denominada Lingüística, filología y literatura. El área de Biblioteconomía y Documentación alcanza un total de 13 revistas, mientras que el resto de áreas contiene un número de revistas bajo.

Tabla 4. Distribución de las revistas según las áreas temáticas asignadas por RESH

\begin{tabular}{|l|c|}
\hline \multicolumn{1}{|c|}{ Área temática } & Núm. revistas \\
\hline Historia & 68 \\
\hline Lingüística, filología y literatura & 43 \\
\hline Bellas Artes & 40 \\
\hline Estudios locales & 31 \\
\hline Interdisciplinares & 23 \\
\hline Biblioteconomía y Documentación & 13 \\
\hline Estudios sobre América Latina & 5 \\
\hline Ciencias jurídicas & 3 \\
\hline Comunicación & 2 \\
\hline Ciencias de la Educación & 2 \\
\hline Filosofía & 2 \\
\hline Geografía & 1 \\
\hline Economía & 1 \\
\hline Ciencias políticas & 1 \\
\hline Sociología & 1 \\
\hline Sin clasificar & 55 \\
\hline & 291 \\
\hline
\end{tabular}

\section{Dispersión de la literatura cientifica. Ley de Bradford}

La ley de Bradford describe la relación cuantitativa entre las revistas y los artículos científicos contenidos en una bibliografía sobre un tema determinado, en este caso el libro antiguo. Se trata de observar cuál es la distribución que presenta el cuerpo de artículos publicados según las revistas donde éstos aparecen, cómo es la dispersión de esos artículos en las revistas y cuál es el 
número de revistas que componen cada una de las zonas de la distribución. En el proceso de concentración, la primera zona llamada núcleo contendrá aquellas revistas más dedicadas al tema de la literatura estudiada.

Para ilustrar la ley se muestra la Figura 2, donde se disponen en el eje de abscisas (eje $X$ ) el logaritmo neperiano del número acumulado de revistas en orden decreciente de productividad, rango $1,2,3, \ldots, r$ y en el eje de ordenadas (eje $Y$ ) el número acumulado de artículos $R(r)$.

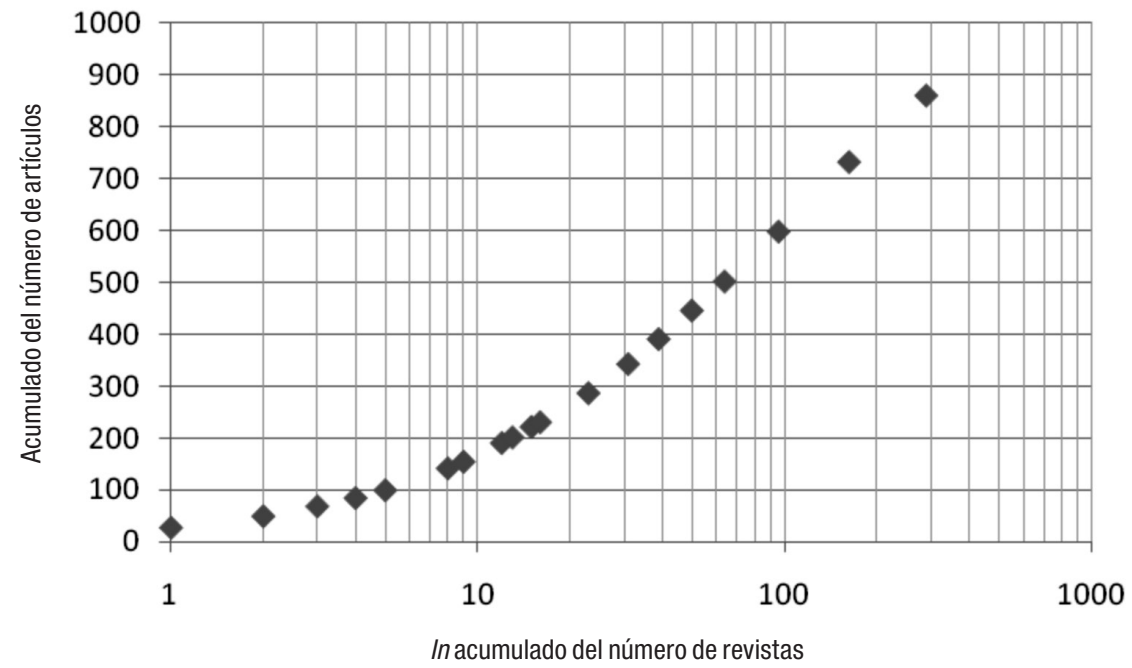

Figura 2. Dispersión de la literatura sobre libros antiguos. Ley de Bradford

La Tabla 5 muestra la dispersión de artículos entre las revistas (columnas 1 y 2). También se incluye el acumulado de las revistas (columna 3), el producto de las dos primeras columnas (columna 4) y el acumulado de los artículos (columna 5).

Tabla 5. Dispersión de la literatura sobre libros antiguos. Ley de Bradford

\begin{tabular}{|c|c|c|c|c|}
\hline Revistas (a) & Artículos (b) & Acum. Revistas & $\mathbf{a}^{*} \mathbf{b}$ & Acum. Artículos \\
\hline 1 & 28 & 1 & 28 & 28 \\
\hline 1 & 23 & 2 & 23 & 51 \\
\hline 1 & 19 & 3 & 19 & 70 \\
\hline 1 & 16 & 4 & 16 & 86 \\
\hline 1 & 15 & 5 & 15 & 101 \\
\hline 3 & 14 & 8 & 42 & 143 \\
\hline 1 & 13 & 9 & 13 & 156 \\
\hline 3 & 12 & 12 & 36 & 192 \\
\hline 1 & 11 & 13 & 11 & 203 \\
\hline
\end{tabular}




\begin{tabular}{|c|c|c|c|c|}
\hline 2 & 10 & 15 & 20 & 223 \\
\hline 1 & 9 & 16 & 9 & 232 \\
\hline 6 & 8 & 22 & 48 & 280 \\
\hline 8 & 7 & 31 & 56 & 336 \\
\hline 10 & 6 & 39 & 60 & 396 \\
\hline 10 & 5 & 50 & 50 & 446 \\
\hline 14 & 4 & 64 & 56 & 502 \\
\hline 32 & 3 & 96 & 96 & 598 \\
\hline 67 & 2 & 163 & 134 & 732 \\
\hline 128 & 1 & 291 & 128 & 860 \\
\hline
\end{tabular}

La metodología aplicada para probar si la distribución de artículos entre las revistas se ajusta a una distribución tipo Bradford fue la propuesta por Egghe (1990). La distribución se ha dividido en tres zonas $(P=3)$ : el número total de artículos es de $860(A=860)$, la revista más productiva cuenta con 28 artículos $\left(Y_{m}=28\right)$ y el número total de revistas es de $291(T=291)$.

Los resultados para tres zonas muestran un multiplicador de Bradford $k=3,68$; el número de revistas del núcleo $r_{0}=16$; el número de artículos en cada zona $y_{0}=286,6$ y los parámetros de la ecuación de Bradford $a=220,4$ y $b=0,167$ respectivamente.

La ecuación de la distribución resultante, calculados los parámetros, es la siguiente:

$$
R(r)=a \times \ln (1+b \times r)=220,4 \times \ln (1+0,167 \times 291)
$$

La distribución de los artículos se ajusta a una distribución tipo Bradford con tres zonas, ya que el resultado de la ecuación es similar al total de artículos de la distribución, lo que indicaría que los parámetros de la ecuación calculados con los datos de la Tabla 5 son correctos. Además, las distintas k calculadas para las distintas zonas son semejantes entre ellas y muy similares al multiplicador de Bradford. El resumen de los cálculos se muestra en el siguiente cuadro.

Resumen de los cálculos

\begin{tabular}{|l|c|c|c|}
\hline \multicolumn{1}{|c|}{ Zonas } & Núm. Revistas & Núm. Artículos & $k$ \\
\hline Núcleo & $r_{0}=16$ & 232 & $\cdots-$ \\
\hline $1^{\mathrm{a}}$ Zona & $r_{1}=59$ & 303 & 3,68 \\
\hline $2^{\mathrm{a}}$ Zona & $r_{2}=216$ & 325 & 3,66 \\
\hline & 291 & 860 & \\
\hline
\end{tabular}

$r_{0}=$ revistas del núcleo; $r_{1}=$ revistas de la primera zona de la distribución; $r_{2}=$ revistas de la segunda zona; $k$ = razón entre el núm. de revistas de una zona y el núm. de la zona anterior. 


\section{Distribución de los documentos por autores}

Al distribuir los documentos por el número de autores se observó que de los 907 documentos había 803 elaborados por un solo autor; 74 documentos por 2 autores; 25 documentos por 3 autores; 3 documentos por 4 autores; 1 documento por 5 autores y 1 documento por 9 autores.

El número de autores diferentes alcanza la cifra de 710. Este número de autores es bastante alto respecto al número total de trabajos producidos. Si se suman los trabajos asignados a cada autor resultan un total de 1052 documentos, lo que hace una media de 1,48 artículos/autor. Por tanto se constata la existencia de una gran dispersión de los documentos entre los autores en la literatura producida sobre el libro antiguo.

Los autores más productivos (con más de 10 trabajos) son:

- José Luis Barrio Moya (Institución de Estudios Complutenses, Alcalá de Henares): 38 trabajos. Son trabajos monográficos de bibliotecas pertenecientes a personajes, fundamentalmente, de los siglos XVII y XVIII.

- Juan José Batalla Rosado (Profesor titular de Historia de América, Universidad Complutense de Madrid): 19 trabajos sobre el estudio de códices, especialmente de las culturas mesoamericanas.

- José Luis Herrera Morillas (Profesor titular de Biblioteconomía y Documentación, Universidad de Extremadura): 14 trabajos centrados en el acceso y difusión al libro antiguo en el contexto de las nuevas tecnologías, y también sobre aspectos artísticos de los libros antiguos (encuadernaciones e ilustraciones).

- Gregorio de Andrés Martínez (académico de la Real Academia de la Historia, miembro del Instituto de Estudios madrileños, fue bibliotecario de la Real Biblioteca del Escorial): 11 trabajos que se centran en el estudio de códices y de varias bibliotecas madrileñas.

- Matilde López Serrano (fue directora de la Real Biblioteca): 11 trabajos. Autora especialista en encuadernación artística y en ilustración de libros antiguos.

\section{Producción cientifica de los autores. Ley de Lotka}

En su forma original la "ley cuadrática inversa de la productividad" dice que el número de autores que producen $\mathrm{x}$ trabajos es proporcional a $1 / x^{2}$.

Actualmente la ley de Lotka se ha reformulado de tal manera que su forma original sería un caso particular de la propia ley: 


$$
y_{x}=c \times x^{-n}
$$

Respecto al caso general se han definido los métodos para calcular los valores de la constante ' $C$ ' y de la pendiente ' $n$ ' (Pao, 1985).

La representación gráfica de los pares de datos $(x, y)$ de la distribución de autores, una vez cortada (par 11, 2), se muestra en la Figura 3. En el eje de abscisas se representan los trabajos publicados por los autores de forma logarítmica, y en el de ordenadas el logaritmo decimal del número de autores.

El resultado es una recta cuya pendiente es de -2.56, ajustada a los pares de puntos con un coeficiente de determinación alto $\left(R^{2}=0,9524\right)$.

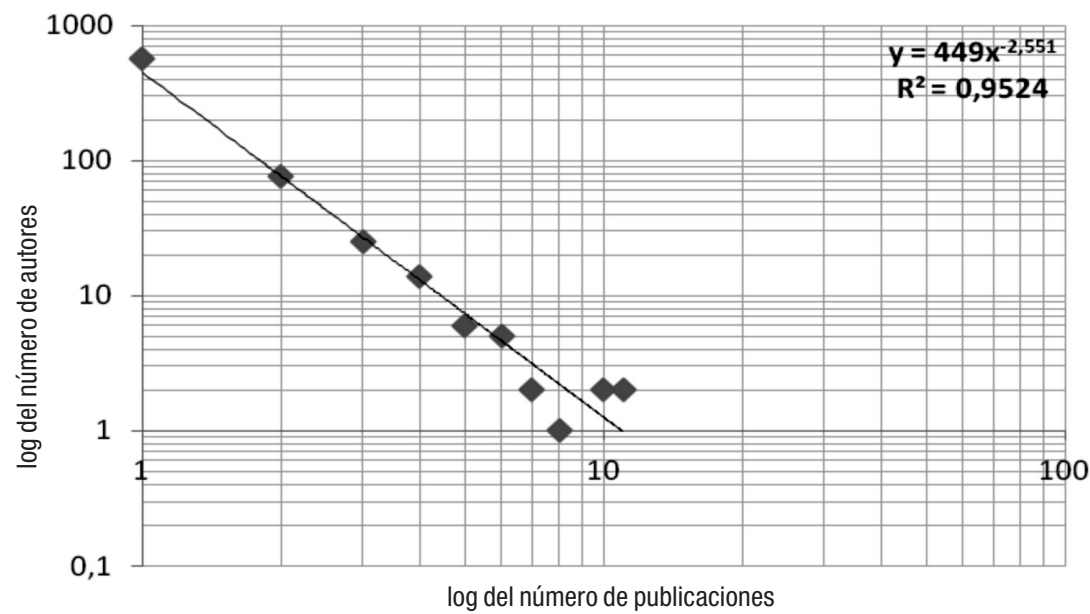

Figura 3. Productividad científica de los autores. Ley de Lotka

Una vez calculada la pendiente se procede a estimar el otro parámetro de la ley de Lotka, la $C$ (porcentaje de autores que publican, teóricamente, un solo trabajo).

En primer lugar se calcula la serie infinita por aproximación:

$$
\sum_{x=1}^{\infty} 1 / x^{n}=\left[\sum_{x=1}^{P-1} \frac{1}{x^{n}}+\frac{1}{(n-1)\left(P^{n}-1\right)}+\frac{1}{2 P^{n}}+\frac{n}{24(P-1)^{n+1}}\right]=1,319
$$

A continuación se obtiene la constante $C$, que se estima como la inversa del valor obtenido de la serie (Pao, 1985):

$$
C=\frac{1}{\sum_{x=1}^{\infty} 1 / x^{n}}=\frac{1}{1,319}=0,7581
$$


Los valores de la Tabla 6 se emplearon para probar (según el test no paramétrico de Kolmogorov-Smirnov) la hipótesis nula $\left(H_{0}\right)$ : "Los datos de la distribución de autores se ajustan a una distribución de tipo Lotka".

La diferencia máxima (Dmax.), resultado de la aplicación del test, es de 0,053 (valor reflejado en la última columna de la Tabla 6).

El valor crítico para un nivel de significación del $99 \%(\alpha=0,01)$ es de 0,061 , tras ser calculado con la siguiente fórmula:

$$
\text { v.c. }=\frac{1,63}{\sqrt{707}}=0,061
$$

Por lo tanto al comparar los dos valores: Dmax. < v.c., se acepta la $H_{0}$ : "Los datos se ajustan a una distribución del tipo Lotka".

Tabla 6. Test de ajuste Kolmogorov-Smirnov de la distribución de autores

\begin{tabular}{|c|c|c|c|c|c|c|}
\hline $\mathbf{x}$ & $\mathbf{y}$ & $\mathbf{y} / \mathbf{\Sigma} \mathbf{y}$ & $\boldsymbol{\Sigma}(\mathbf{y} / \mathbf{\Sigma} \mathbf{y})$ & $\mathbf{f e}$ & $\boldsymbol{\Sigma} \mathbf{f e}$ & Dmax. \\
\hline 1 & 574 & 0,8119 & 0,8119 & 0,7581 & 0,7581 & 0,0538 \\
\hline 2 & 76 & 0,1075 & 0,9194 & 0,1285 & 0,8866 & 0,0328 \\
\hline 3 & 25 & 0,0354 & 0,9548 & 0,0455 & 0,9321 & 0,0227 \\
\hline 4 & 14 & 0,0198 & 0,9746 & 0,0217 & 0,9538 & 0,0208 \\
\hline 5 & 6 & 0,0085 & 0,9831 & 0,0123 & 0,9661 & 0,0170 \\
\hline 6 & 5 & 0,0071 & 0,9902 & 0,0077 & 0,9738 & 0,0164 \\
\hline 7 & 2 & 0,0028 & 0,9930 & 0,0052 & 0,9790 & 0,0140 \\
\hline 8 & 1 & 0,0014 & 0,9944 & 0,0036 & 0,9826 & 0,0118 \\
\hline 10 & 2 & 0,0028 & 0,9972 & 0,0020 & 0,9846 & 0,0126 \\
\hline 11 & 2 & 0,0028 & 1 & 0,0016 & 0,9862 & 0,0138 \\
\hline & 707 & 1 & & & & \\
\hline
\end{tabular}

\section{Filiación de los autores}

Se han localizado un total de 160 lugares de trabajo. Y se han considerado como lugares de afiliación más productivos aquellas instituciones en las que trabajan más de 18 autores.

Destaca la Universidad Complutense de Madrid con 96 autores, mostrando una posición privilegiada respecto al resto de instituciones.

Otras instituciones que también destacan son: el Instituto de Estudios Complutenses de Alcalá de Henares con 34 autores; la Universidad de Barcelona con 22 autores; la Universidad de Valencia con 22 autores; la Universidad de Sevilla con 20 autores; la Universidad de Salamanca con 19 autores; la Universidad de Zaragoza con 16 autores; la Universidad Nacional de Educación a Distancia con 14 autores; la Universidad de Valladolid con 14 autores y la Universidad de Murcia con 14 autores. 


\section{Conclusiones}

La base de datos ISOC es una interesante e importante fuente para el estudio del libro antiguo por el notable número de trabajos localizados, que nos ha llevado a precisar las revistas científicas especializadas y afines en las que se suelen publicar los trabajos, y que pueden ser objeto de otros estudios cuyos temas completen este artículo.

Los trabajos sobre libros antiguos se han publicado en una amplia variedad de revistas pertenecientes a diversas áreas temáticas dentro de las $\mathrm{Hu}$ manidades y las Ciencias sociales. Las revistas están englobadas en más de 16 áreas temáticas (siguiendo la clasificación de RESH). Las que presentan un número significativo de trabajos (más de 10) son 6 áreas (Historia; Lingüística, filología y literatura; Bellas Artes; Estudios locales; Intersdisciplinares; Biblioteconomía y Documentación).

La información anterior ratifica, desde el punto de vista bibliométrico, una destacada dispersión en las revistas y una baja productividad. En el caso de los autores pasa algo parecido.

Los trabajos más antiguos relacionados con el libro antiguo se han publicado en revistas de Historia, Lingüística, Estudios locales, etc. Desde hace pocos años las revistas de Biblioteconomía y Documentación han mejorado su representación. Los trabajos de los últimos años aparecen tanto en unas como en otras, si bien los estudios dentro de las revistas de Biblioteconomía y Documentación están creciendo.

Como resultado de esta investigación se tiene una visión más detallada del panorama investigador relacionado con el libro antiguo en España: trabajos, revistas, autores, lugares de investigación, etc. La Universidad Complutense de Madrid refleja una contribución destacada que se refleja, fundamentalmente, en la afiliación de los autores o en algunas de las revistas con más trabajos y que están vinculadas a esta universidad.

Este trabajo permite evaluar y tener una visión más exacta sobre la base de datos ISOC: se trata de una base de datos muy completa por la cobertura cronológica y la variedad de disciplinas que abarca. Sin embargo, se han detectado algunas carencias en la información de los registros; la más significativa es que bastantes registros carecen de los campos resumen y descriptores, aunque algunos ofrecen el enlace al texto completo del documento, donde sí aparecen dichos elementos. Finalmente, los resultados abren algunas inquietudes que se podrían resolver en posteriores investigaciones, sobre todo debido a la gran dispersión de áreas temáticas de las revistas en las que aparecen publicados los trabajos recopilados. Habría que precisar e indagar en la diferente metodología investigadora que diferencia a unos de otros, según las áreas. 


\section{BiBLIOGRAFÍA}

Aguirre, J. L., "Bibliometría elemental sobre literatura española de los siglos de oro", en Boletín de la Sociedad Castellonense de Cultura, v. 63 (3), pp. 331-341, 1987.

Berger, Ph., "La evolución de la producción editorial española entre 1501 y 1520", en El libro antiguo español: actas del Primer Coloquio Internacional (Madrid, 18 al 20 de diciembre de 1986), pp. 63-72, Salamanca: Ediciones Universidad de Salamanca, 1988.

Delgado López-Cozar, E.; Cordón García, J. A., El libro: creación, producción y consumo en la Granada del siglo XVII, Granada: Universidad de Granada, 1990.

Egghe, L., "Applications of the theory of Bradford's law to the calculation of Leimkuhler's law and to the completion of bibliographies", en Journal of the American Society for Information Science, v. 41 (7), pp. 469-492, 1990, disponible en: http://www.ub.edu/ $\mathrm{bid} / 25 /$ estivill2.htm [consultado el 24 marzo 2012].

Fernández Dueñas, A. et al., "La producción médico editorial cordobesa en el Barroco: análisis, revisión y comentarios", en Boletín de la Real Academia de Córdoba de Ciencias, Bellas Letras y Nobles Artes, v. 55 (106), pp. 347-357, 1984.

García Cuadrado, A., "Una imprenta murciana del siglo XVIII: aproximación a su producción bibliográfica (1759-1780)", en Escavy Zamora, R. (coord.), Amica Verba: in honorem Prof. Antonio Roldán Pérez, vol. 1, Murcia: Universidad de Murcia, Servicio de publicaciones, pp. 299-316, 2005.

Gil Fernández, L., "La producción editorial de signo humanístico en la época de los Reyes Católicos", en Silva. Estudios de Humanismo y Tradición Clásica, v. 3, pp. 203-222, 2004.

Guillén Grima, F. et al., "Análisis bibliométrico comparativo de la producción sobre Salud pública con el resto de la producción científica médica en el siglo XVIII", en VIII Congreso Nacional de Historia de la Medicina. Libro de actas, v. 3, pp. 1197-1213, 1998. Murcia: Departamento de Historia de la Medicina.

Herrero Pascual, C., "Análisis documental y bibliométrico de los libros del siglo XVI de la Biblioteca de los Obispos (Murcia)", en Revista General de Información y Documentación, v. 7 (2), pp. 141166, 1997.

Liñán Maza, M. del C., "La producción bibliográfica andaluza del siglo XIX en la Biblioteca de la Facultad de Veterinaria de Córdoba”, en S. Hernández Rodríguez (ed.), In memoriam al profesor doctor D. Francisco de Paula Martínez Gómez. Córdoba: Universidad de Córdoba, Servicio de publicaciones, pp. 951-968, 1992.

Mansilla Legorburu, M. E., Catalogación, estudio estadístico y bibliométrico de los libros de interés bistórico-farmacéutico que se encuentran en la biblioteca central de la Universidad literaria de Valencia, (Tesis doctoral, Universidad de Valencia), 1984. 
Olagüe de Ros, G., La literatura médica sobre epilepsia: Siglos XVI-XIX: Análisis bibliométrico. Valencia: Cátedra e Instituto de Historia de la Medicina, 1976.

Pao, M. L., "Lotka's law: a testing procedure", en Information Processing E Management, v. 21 (4), pp. 305-320, 1985.

Pardo Tomás, J., "La producción impresa de libros científicos en la Corona de Aragón durante el siglo XVI", en Serrano Martí, E.; Sarasa Sánchez, E. (coord.), La Corona de Aragón y el Mediterráneo: siglos XV-XVI, Zaragoza: Institución Fernando el Católico, pp. 231 268, 1997.

Puche Azarín, P., La literatura médica española del siglo XVIII. Inventario, análisis bibliométrico y estudio semántico, Valencia: Cátedra de Historia de la Medicina (Tesis de Licenciatura), 1975.

Pulgarín Guerrero, A.; Herrera Morillas, J. L.; Marroquín Martínez, L., "Estudio bibliométrico de la Biblioteca de la Real Sociedad Económica Extremeña de Amigos del País. Parte I, colección de los siglos XVI-XVIII", en Revista General de Información y Documentación, v. 17 (2), pp. 183-208, 2007.

Pulgarín, A.; Herrera Morillas, J. L., “Aplicación de la Bibliometría al estudio del fondo antiguo: estado de la cuestión y vías de investigación”, en IV Encontro Ibérico EDICIC 2009, Coimbra 18-20 de noviembre de 2009, v.2, pp. 273-286. Coimbra: Universidad, 2009.

Sáez Gómez, J. M. Marset Campos, P., "La producción científico-médica murciana en el siglo XVIII", en Llull. Boletín de la Sociedad Española de Historia de las Ciencias, v. 16 (31), pp. 537-548, 1993.

Sánchez Serena, M. A., La literatura farmacéutica española, 1494-1800. Inventario, análisis bibliométrico y estudio semántico (Tesis Doctoral, Universidad de Valencia), 1978.

Sillero Sarasua, M. P., La biblioteca de Philipp Hauser. Análisis bibliométrico e inventario (Tesis doctoral, Universidad de Sevilla), 1992.

Suárez de la Torre, L., La producción de libros, revistas, periódicos y folletos en el siglo XIX, en Clark de Lara, B.; Speckman Guerra, E. (coords.), La república de las letras: asomos a la cultura escrita del México decimonónico, v. 2, México: Universidad Nacional Autónoma de México, pp. 9-28, 2005. 